\title{
Structure and reentrant percolation in an inverse patchy colloidal system
}

\author{
J. L. B. de Araújo, ${ }^{1, *}$ F. F. Munarin,${ }^{2, \dagger}$ G. A. Farias, ${ }^{1, \dagger}$ F. M. Peeters, ${ }^{3, \S}$ and W. P. Ferreira ${ }^{1, \mathbb{d}}$ \\ ${ }^{1}$ Department of Physics, Federal University of Ceará, Fortaleza, Ceará 60.455-970, Brazil \\ ${ }^{2}$ Department of Academic and Technological Integration, Federal University of Ceará, Fortaleza, Ceará 60.455-900, Brazil \\ ${ }^{3}$ Department of Physics, University of Antwerp, Groenenborgerlaan 171,B-2020 Antwerpen, Belgium
}

(Received 7 November 2016; published 26 June 2017)

\begin{abstract}
Two-dimensional systems of inverse patchy colloids modeled as disks with a central charge and having their surface decorated with oppositely pointlike charged patches are investigated using molecular dynamics simulations. The self-assembly of the patchy colloids leads to diverse ground state configurations ranging from crystalline arrangements of monomers to linear clusters, ramified linear clusters and to percolated configurations. Two structural phase diagrams are constructed: (1) as a function of the net charge and area fraction, and (2) as a function of the net charge and the range of the pair interaction potential. An interesting reentrant percolation transition is obtained as a function of the net charge of the colloids. We identify distinct mechanisms that lead to the percolation transition.
\end{abstract}

DOI: 10.1103/PhysRevE.95.062606

\section{INTRODUCTION}

Colloidal particles have attracted a lot of interest in soft matter and material science due to their broad potential applicability in, e.g., biomaterials, catalytic supports, and lightweight structural materials [1-3]. Spherical colloids are most popular due to their convenient preparation providing uniform particle size and shape [4]. New synthesis routes for colloids have given the possibility to create functional materials with enhanced complexity and functionality [4-6]. In general, the number of different structures formed by spherical particles (with isotropic interaction) is limited. In order to expand the possibilities of new building blocks, the use of particles with heterogeneous shapes and interactions have been proposed [7-10]. The phase behavior of colloidal particles with heterogeneously patterned surfaces, called patchy particles, has been a very active field of research [5,11-14] not only from a fundamental point of view, but also because it allows the synthesis of exotic building blocks for the self-assembly of new materials $[12,15,16]$.

Anisotropic patchy models have received interest as a model system, e.g., in the context of protein crystallization [17-20]. In general, proteins are hard to crystallize. The crystal functionality of a given protein can be studied through the analysis of its structure in large crystals using high-resolution $\mathrm{x}$-ray diffraction [21]. Theoretical studies are very useful to predict when crystallization occurs. In this context, some contributions have been made through theoretical models with isotropic potentials. However, it is known that protein interactions are short ranged and highly anisotropic and consequently such models will, at some point, break down [22].

Much effort is currently devoted to the development of sophisticated techniques for the synthesis of anisotropic

\footnotetext{
*jlbaraujo@fisica.ufc.br

${ }^{\dagger}$ munarin@fisica.ufc.br

${ }^{\ddagger}$ gil@ fisica.ufc.br

§francois.peeters@uantwerpen.be

Ipaiva@fisica.ufc.br
}

colloidal particles. These techniques come from a combination of diverse fields providing a powerful arsenal for the fabrication of new building blocks [23-26]. Patchy particles with interactions given by electrostatic repulsion in the middle and hydrophobic attraction at the poles were fabricated recently by Chen et al. [27], which used them as building blocks to obtain a complex predetermined colloidal open kagome structure. Diverse experimental [28,29], theoretical [30-32], and computer simulation studies [33-35] showed that aggregates can be obtained from the competition between a screened electrostatic repulsion and a short-range attraction between the colloids. Besides, the shape and size of such aggregates depend on the balance between repulsion and attraction [36,37], as observed in protein solutions at the limit of low salt concentration [28,38]. Very recently, Rikkert et al. [39] investigated and quantified the effects of the $p \mathrm{H}$ and salt concentration on the charge regulation of the bacteriophage PP7 capsid. These effects are found to be extremely important, introducing qualitative changes in the charge state of the capsid such as a transition from net-positive to net-negative charge depending on the $p \mathrm{H}$ of the solution.

Introduced by Bianchi et al. [40], inverse patchy colloids (IPCs) are colloidal particles whose anisotropic interaction originates from the presence of oppositely charged regions on the surface of a uniformly charged colloid. In contrast to conventional patchy colloids, where the patches exhibit short-range attractive interaction, the interaction between the charged patches is repulsive, with the added possibility to tune the range of the interaction, through the screening of the Coulomb interaction [40] by the ions in the solution [41]. The uniform charge of an IPC is modeled by a central charge with opposite sign with respect to the ones in the patches. As a consequence, the interaction between two IPCs can be attractive or repulsive according to the relative orientation between them. The system with IPCs is thus characterized by a nontrivial competition between attractive and repulsive directional interactions.

Noya et al. studied the phase behavior of colloidal particles with two charged patches in three dimensions [42]. It was shown that the system exhibits an unusual equilibrium phase diagram characterized by a broad region where a novel 
structure composed of parallel colloidal monolayers is stable. On the other hand, an investigation of IPCs in two dimensions showed different cluster sizes with tunable spatial and orientational order [43]. In this study, the electrostatic interaction between the colloids and the substrate was included. It is interesting to notice that previous studies of IPCs in two $[43,44]$ and three dimensions considered mostly neutral IPCs, i.e., the absolute value of the charge in the center is equal to the sum of those in the patches. Recently, Peng et al. presented a method to fabricate patchy particles consisting of different materials in the center and the poles [4]. The authors used seeded emulsion polymerization to fabricate a nearly monodisperse system of linear-trimeric particles, i.e., particles with opposing lobes at the surface. Note that different materials in a given solvent may result in colloids which have parts charged with opposite signs. Besides that, van Oostrum et al. [45] created IPCs with center charge and opposite charged caps at the surface of each particle. The different regions of particles (center or caps) could be distinguished using fluorescence microscopy.

In this work, we present a systematic computer simulation study of a two-dimensional system of IPCs composed of two polar patches as a function of the net charge of IPCs which may be related to the effects of the $p \mathrm{H}$ and/or salt concentration of the solvent in which the particles are immersed [39]. We discover a rich set of characteristic configurations for distinct values of the net charge. The dependence of the critical percolation density, the percolated structures, and the mechanisms of percolation for distinct net charges are also described, highlighting a reentrant percolation phase which is observed by changing the net charge of the IPC for fixed density. The shape and microstructure of the different configurations are discussed.

The paper is organized as follows. Our model system is presented in Sec. II. The results and discussions are presented in Secs. III- VI and our conclusions are given in Sec. VII.

\section{MODEL}

We consider a two-dimensional system in which a given IPC is allowed to occupy any position in the two-dimensional (2D) plane. Each IPC is a hard disk with mass $m_{0}$, diameter $\sigma$, and a central positive pointlike charge $Q$ and two patches, each with negative pointlike charge $-q$, located at the surface of the disk and diametrically opposite. The diametral line connecting the two patches defines the main axis of a given IPC (Fig. 1). The interparticle potential of our system consists of a Yukawa potential plus a hard-disk repulsion term. The full pair interaction potential is given by

$$
U_{i j}=U_{i j}^{\mathrm{HD}}+U_{i j}^{Y}
$$

with the hard-disk potential

$$
U_{i j}^{\mathrm{HD}}=h\left(\frac{\sigma}{r_{i j}}\right)^{12},
$$

where $h$ is a constant of energy and $r_{i j}$ is the separation between the centers of particles $i$ and $j$. The last term in Eq. (1) is the Yukawa pair interaction potential between the charges of (a)

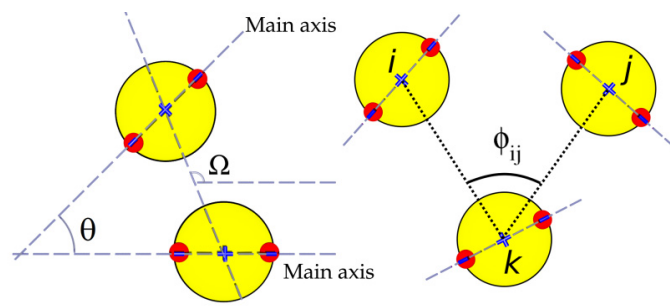

(b)
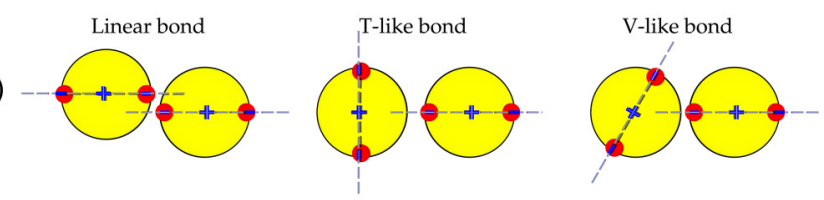

(c)

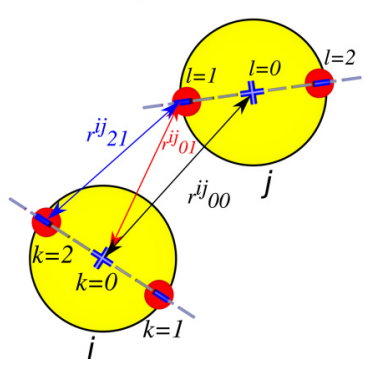

FIG. 1. Schematic view of the model (a) and the different types of bonds (b). Blue crosses refer to the positive charge in the center of the colloid and the red dots are the negative patches at the surface. $\theta$ is the angle between the main axis of two IPCs. The angle $\phi$ is the angle between the lines connecting the centers of two IPCs $i$ and $j$ with the central particle $k$ as the vertex, where $i$ and $j$ are neighbors of $k$. The angle $\Omega$ is the angle between the line connecting the centers of two IPCs $i$ and $j$ and the $x$ axis. In (c) we show some examples of distances $r_{k l}^{i j}$.

different colloids $i$ and $j$,

$$
U_{i j}^{Y}=\sum_{k=0}^{2} \sum_{l=0}^{2} \frac{Z_{k} Z_{l}}{\epsilon} \frac{\exp \left(-\kappa r_{k l}^{i j}\right)}{r_{k l}^{i j}}
$$

where $Z_{0}=+Q, Z_{1}=Z_{2}=-q, \kappa$ is the inverse of the Debye screening length, and $\epsilon$ is the medium permittivity. The terms $r_{k l}^{i j}$ are the distances between the different parts of each particle $i$ and $j$ [see Fig. 1(c)]. The indexes $k$ and $l$ represent a tag for each part of the particle. $k=0$ and $k \neq 0$ (or $l=0$ and $l \neq 0$ ) represent the indexes for the center and the two patches of each particle, respectively. In order to reveal important parameters which characterize the system, we rewrite the energy $U_{i j}$ in a dimensionless $\left(u_{i j}\right)$ form by making use of the following variable transformations: $U_{i j}^{Y}=$ $E_{0} u_{i j}^{Y}, U_{i j}^{\mathrm{HD}}=E_{0} u_{i j}^{H D}, r_{i j} \rightarrow r_{i j} \sigma, \kappa \rightarrow \kappa / \sigma$, where $E_{0}$ is the unit of energy. Moreover, the net charge of each particle is given by $C=Q-2 q$ and we can normalize it in terms of $q$ $(C \rightarrow q C$ ), with $\alpha=Q / q$ being the ratio between the charge at the center and the charge of each patch. Thus, the net charge of each particle is $C=\alpha-2$. The pair interaction potential is given by

$$
u_{i, j}^{\mathrm{HD}}=\frac{h}{E_{0}}\left(\frac{1}{r_{i j}}\right)^{12}
$$


and

$$
U_{i j}^{Y}=\frac{q^{2}}{\epsilon \sigma E_{0}} \sum_{k=0}^{2} \sum_{l=0}^{2} Z_{k}^{\prime} Z_{l}^{\prime} \frac{\exp \left(-\kappa r_{k l}^{i j}\right)}{r_{k l}^{i j}},
$$

where $Z_{0}^{\prime}=\alpha$ and $Z_{1}^{\prime}=Z_{2}^{\prime}=-1$. We fixed $h=q^{2} / \epsilon \sigma=$ $E_{0}$. Thus, the unit of time is written in terms of $t_{0}=$ $\sqrt{m_{0} \sigma^{2} / 2 E_{0}}$. The ground state will depend on the number of particles $N$ per computational unit cell (i.e., the area fraction $\eta$ ), the ratio between charges $\alpha$, and the inverse Debye screening length $\kappa$. In this way, the interaction energy is effectively related to the net charge $(C)$ of a given IPC when a patchy particle is neutral $(C=0)$ or overcharged $(C \neq 0)$.

The simulations are conducted within a molecular dynamics [46] protocol (MD) with a periodic repeated simulation box of area $A=L^{2}$. We cut the interparticle potential at $r_{c}=20 \sigma$ where the interaction energy between two IPCs, regardless of $\theta, \Omega$, and $C$, approaches $E_{\max }\left(r_{c}\right) \sim 10^{-9} E_{0}$ in the case $\kappa=1$. The equations of motion are integrated using the velocity Verlet algorithm with a time step of $0.01 t_{0}$. Temperature $T$ is fixed by means of the Berendsen thermostat with a time constant of 10 ( $N V T$ ensemble). The results presented here are obtained at temperature $T / T_{0}=10^{-3}$, which is constant during all simulations with $T_{0}=E_{0} / k_{b}$ and $k_{b}$ the Boltzmann constant. The area fraction is defined as $\eta=N \pi \sigma^{2} / 4 L^{2}$, which represents the fraction of the simulation box occupied by the IPCs. Different values of the area fractions are obtained by using a simulation box with constant size and considering different numbers of particles $(N=600-2000)$. Typically, the equilibrium structures are obtained after $10^{6}$ time steps, which are enough to stabilize the total energy of the system for most of the cases. After equilibration, we run additional $2 \times 10^{6}$ time steps in order to average the quantities of interest (cluster size distribution, gyration radius, etc.). For some specific cases, it is not easy to find the stable configuration due to the large number of metastable states (local minima) in the configuration space. A standard procedure to test if the minimum energy configuration was indeed reached is to heat up the system and cool it down at different rates. We then compare the energy of the obtained configurations. Such a procedure is repeated several times in our simulations in addition to the use of different initial configurations. The cluster phases are defined by a visual and geometric criterium. In all obtained configurations if separation between neighbor particles in a cluster is less than $r_{\text {bond }} \approx 1.3 \sigma$ we consider a pair of particles as bonded. The reason is that for such a separation the interparticle distance is in the attractive part of the potential and corresponds to the area between the first two peaks in the radial distribution function (see Sec. IV).

\section{PHASE DIAGRAM}

We show first that some interesting information can be obtained from the functional form of the pair-interaction potential when minimized with respect to the relative orientation between IPCs (angles $\theta$ and $\Omega$ in Fig. 1). For example, for neutral IPCs $(C=0)$ the pair-interaction potential is attractive (see Fig. 2), which favors aggregation of particles into clusters. As will be shown, this is indeed observed in the many-body case. The dependence of the pair-interaction potential with

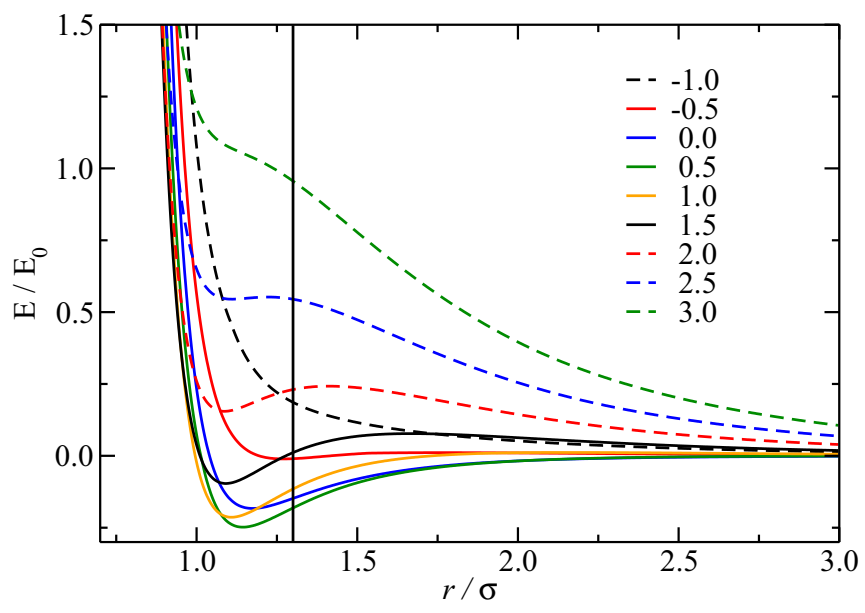

FIG. 2. The lowest interaction potential energy between two particles for different values of the net charge $C$, i.e., minimized with respect to the particle orientation angles $\theta$ and $\Omega$ (see Fig 1). The solid lines indicate the presence of a global minimum energy for $r \leqslant r_{\text {bond. }}$. Dashed lines are for the cases that the lowest interaction energy is found for $r \rightarrow \infty$.

respect to the separation between IPCs for different values of the net charge $C$ is shown in Fig. 2. The curves correspond to the lowest energy between two IPCs from all possible relative orientations; in other words, angles $\theta$ and $\Omega$ shown in Fig. 1, where $\theta$ is the angle between the main axis of two IPCs and $\Omega$ is the angle between the line connecting the centers of two particles and the $x$ axis. To analyze the structures found, we defined the angle $\phi$ [Fig. 1(b)] as a triplet angle calculated as

$$
\phi_{i, j}=\cos ^{-1}\left(\frac{\overrightarrow{R_{i, k}} \cdot \overrightarrow{R_{j, k}}}{\left|\overrightarrow{R_{i, k}}\right|\left|\overrightarrow{R_{j, k}}\right|}\right) .
$$

In general, due to the nonhomogeneous charge distribution over each IPC, the interaction is not symmetric with respect to $C$. For negative net charge, the excess of charge is on the surface of the particle, favoring repulsion between distinct IPCs. For positive net charge, the excess is on the center of the particle, favoring attractive interaction. For example, the pair interaction potential for $C=-1$ is repulsive, while for $C=1$ it presents an attractive short-range well and becomes almost zero for $r \gtrsim 1.75$. As a consequence, we expect that in the many-body case with $C=-1$ no clustering will be observed. Indeed, for $C=-1$ the IPCs self-assemble as a set of monomers, where for sufficiently small temperature $\left(T / T_{0} \sim 10^{-6}\right)$ the IPCs are arranged in a configuration with both translational and orientational order. On the other hand, in the many-body case with $C=1$ no monomers are found. Instead, the IPCs are arranged in linear clusters. As can be seen in Fig. 2, the pair interaction potential becomes repulsive for large enough positive $C \geqslant 3$, indicating that in the many-body case monomers should again be observed.

We stress here that the curves presented in Fig. 2 are the ones which minimize the pair interaction energy with respect to the angles $\theta$ and $\Omega$. Interestingly, out of those values of $\theta$ and $\Omega$, a rather distinct qualitative behavior may be observed for $C=3$ in spite of the minimum pair-interaction potential presented in Fig. 2 being repulsive for any $r$; an attractive pair 


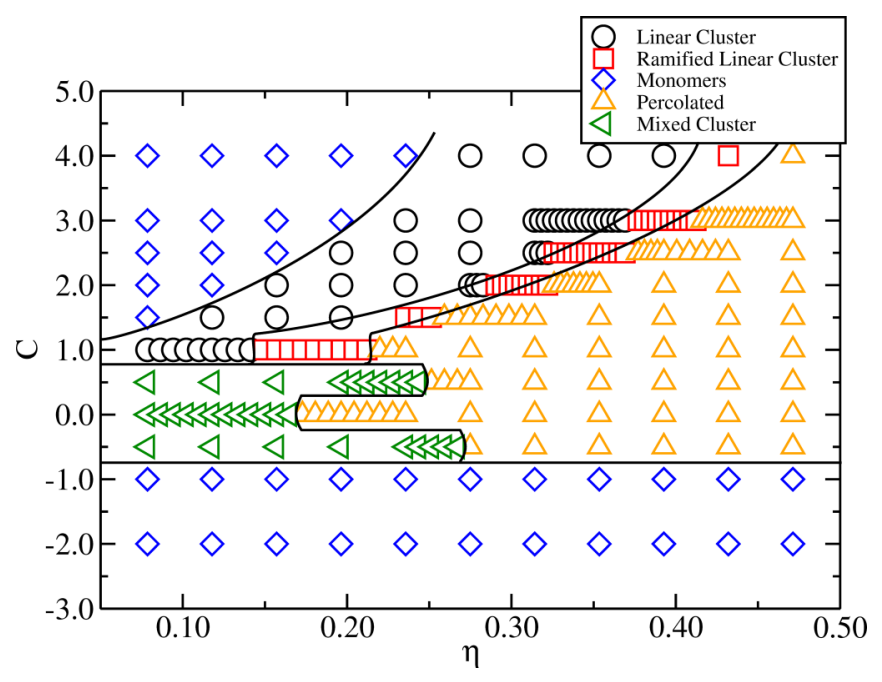

FIG. 3. Structural phase diagram in the plane of net charge $(C)$ of each IPC and area fraction of the system $(\eta)$. Symbols represent different structures: $\diamond$, monomers; $\triangleleft$, mixed clusters; $\circ$, linear clusters; $\square$, ramified-linear clusters; and $\triangle$, percolated clusters. Solid lines are guides to the eyes separating the different phases.

interaction is still possible for some specific values of $\theta$ and $\Omega$. Notice that in the many-body case, due to the complex interaction among the IPCs, the relative orientations between neighbor particles do not always correspond to the one which minimizes the pair interaction energy.

In general, a short-range attractive well competes with a long-range repulsive tail, with the magnitude of both being dependent on $C$. In addition, the presence of a global minimum at finite $r$ indicates that clusters may appear. In the many-body case clusters are found in a finite interval of area fraction and net charge.

From our systematic MD simulations we find an interesting set of stable configurations, which are summarized in a $(C, \eta)$ structural phase diagram (Fig. 3). The set of obtained configurations consists of monomers (blue diamond), linear clusters (black circle), ramified-linear clusters (red square), mixed cluster (green left-triangle), and percolated configurations (yellow up-triangle). Linear clusters are characterized by a sequence of IPCs with main axis aligned along the cluster $\left(\theta=0^{\circ}\right.$ and $\phi=135^{\circ}$ in Fig. 1). When such linear clusters join one another or when one or more lines start to grow from a given one, a ramified-linear cluster is formed. For mixed clusters the majority of the connections between IPCs is such that neighbor bonded particles have their main axis perpendicular to each other. However, there are also some other small clusters in which the main axis of the particles have an angle smaller than $90^{\circ}$. The monomers to linear clusters transitions $(C>1)$ can be found by analyzing the average interparticle separation $R_{1}$ given by the position of the first peak of radial distribution $g(r)$. Usually, for bidimensional systems with repulsive interaction, $R_{1}$ depends on the area fraction $\eta$ following a power law of the form $R_{1}(\eta) / \sigma \sim \eta^{-1 / 2}$. Thus, when one cluster is found, a little peak in $g(r)$ is observed for $r \leqslant r_{\text {bond }}<R_{1}(\eta)$.

From the obtained clusters we basically observe three types of bonds between IPCs, namely, linear, T-like, and V-like bonds. The latter is mainly observed in the ramified linear clusters, where it corresponds to the connection between distinct linear clusters (or a new branch in a linear cluster) or in small clusters (e.g., three particles) in the mixed cluster phase. A schematic view of the distinct types of bonds is shown in Fig. 1.

Examples of typical configurations in the different regions of the phase diagram shown in Fig. 3 are presented in Fig. 4, namely, monomers [Figs. 4(a) and 4(h)], mixed clusters [Figs. 4(b) and 4(d)], percolated structures [Figs. 4(c) and 4(e)], ramified-linear clusters [Fig. 4(f)], and linear clusters [Fig. 4(g)]. The configurations presented in Fig. 4 have the same area fraction $\eta=0.235$ and different net charges. In general, by changing the net charge of each IPC we are able to modify the microstructure of the configurations. For example, for $C=-1$ [Fig. 4(a)], the total energy per particle is repulsive, and only monomers are observed. In this case, we find that the monomers are arranged in a square lattice, for $\eta \gtrsim 0.235$, with defects due to the relatively small value of the potential energy as compared to the kinetic energy. In Fig. 5(a) we present the probability distribution of the triplet angle $\phi$ defined in Eq. (6) for $C=-1$ and different area fractions. For $\eta \gtrsim 0.235$, the pronounced peaks near to $\phi=90^{\circ}$ and $\phi=180^{\circ}$ indicate the presence of a square lattice structure. For lower temperature $T / T_{0}=10^{-6}$ (not shown), the number of defects is drastically reduced and a square lattice becomes clear with both translational and orientational order, i.e., the IPCs in neighboring lattice sites have their main axes perpendicular to each other. For $0.078 \lesssim \eta<0.235$ we observe a mixing of triangular and square lattices. For small area fraction, $\eta \lesssim 0.078$, the IPCs self-assemble in a triangular lattice. Normally, in the zero temperature limit a classical system of particles with repulsive interaction will assemble in a periodic structure that minimizes the potential energy. It is well known that the triangular structure is the only stable arrangement in $2 \mathrm{D}$ for particles with repulsive radial symmetric pair-interaction potential [47]. Besides that, Malescio and Pellicane [48] showed that it is possible to find other structures in 2D with isotropic repulsive interaction. The increase of density can promote the transition from the triangular lattice to the stripe phase. In our case, for $C=-1.0$ and low temperature, it is possible to observe the transition from triangular to square lattice with increasing density. However, the different arrangements observed here for $C=-1$, especially the square lattice for high enough area fraction, is a consequence of the nonhomogeneous charge distribution in each IPC. More generally, monomers are observed for $C \leqslant-1$. For neutral IPCs $(C=0)$ the system always self-organizes in a single cluster mostly with T-like bonds. The larger the area fraction $\eta$, the larger the size of the single cluster. For $\eta \approx 0.16$ the single cluster becomes percolated. On the other hand, for a small, but nonzero, net charge, e.g., $C=-0.5$ and $C=0.5$, the IPCs self-assemble as a set of small clusters with mostly T-like bonds [Figs. 4(b) and $4(\mathrm{~d})$ ]. For $C=1$ the system is already percolated for $\eta=0.235$. In this case, the IPCs are mostly bonded to one another through linear bonds. For $C>1$, the total energy per particle is positive (repulsive). The larger $C$ the larger the total energy per particle indicating that repulsion among IPCs is stronger. This can be seen in Figs. 4(f)-4(h) where 
(a) $\diamond$

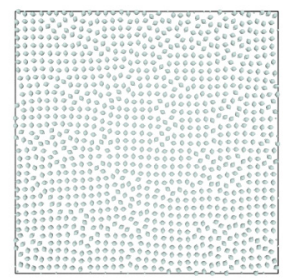

(e) $\diamond$

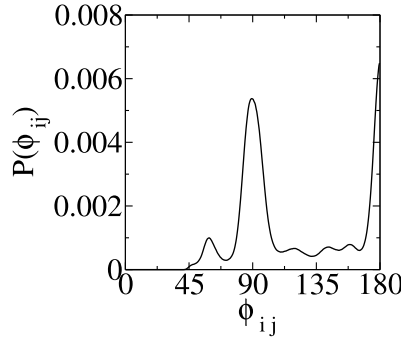

(i) $\triangle$

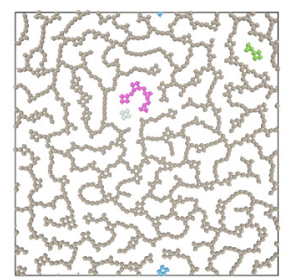

(m) $\triangle$

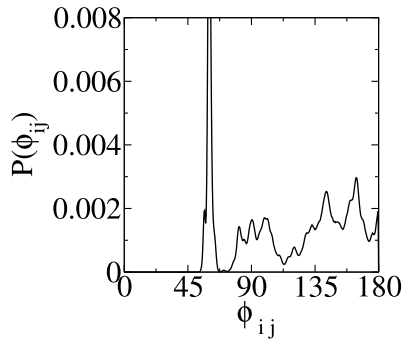

(b) $\triangleleft$

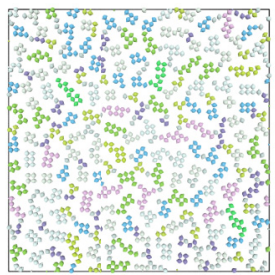

(f) $\triangleleft$

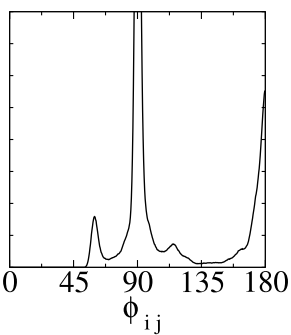

(j)

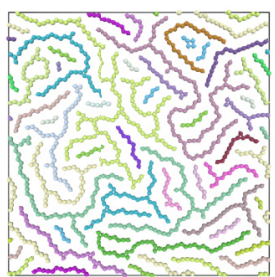

(n)

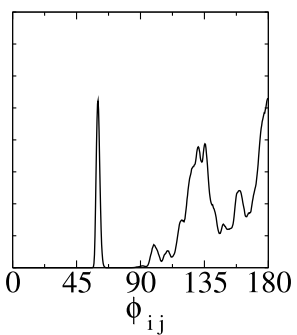

(c) $\triangle$

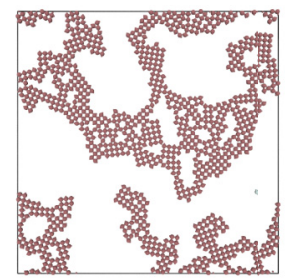

(g) $\triangle$

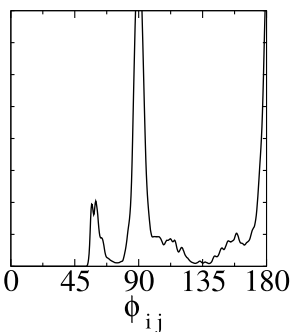

(k) $\mathrm{O}$

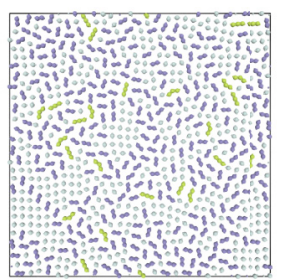

(o) $\bigcirc$

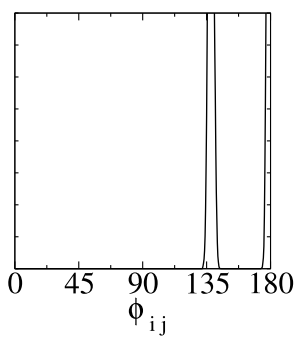

(d) $\triangleleft$

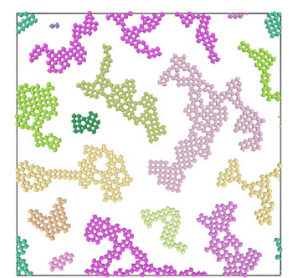

(h) $\triangleleft$

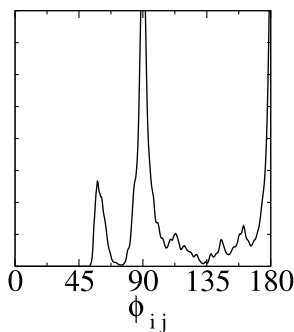

(l) $\diamond$

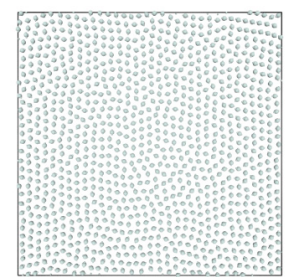

(p) $\diamond$

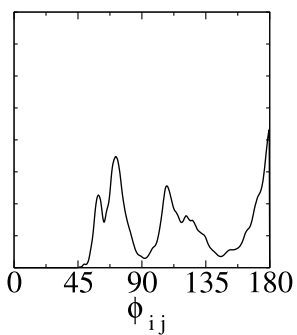

FIG. 4. Representative configurations observed in the $(C, \eta)$ phase diagram for area fraction $\eta=0.235$ and different net charge (a) $C=-1.0$, (b) $C=-0.5$, (c) $C=0.0$, (d) $C=0.5$, (i) $C=1.0$, (j) $C=1.5$, (k) $C=3.0$, and (1) $C=4.0$. Different colors represent clusters of different sizes. The symbols above each figure refer to the configuration in the phase diagram of Fig. 3. From (e)-(h) and (m)-(p) are shown the distributions of the triplet angle $\phi_{i, j}$ defined in Eq. (6) (see Fig. 1) for the structures (a)-(d) and (i)-(1), respectively. In all the cases $P\left(\phi_{i, j}\right)$ is a normalized distribution.

the size of the linear clusters decreases with increasing $C$ (for $\eta=0.235$ ). For a given $C>1$ we always observe the same sequence of configurations (monomers, linear clusters, ramified-linear clusters, and percolated) as the area fraction is increased (Fig. 3).

Interestingly the monomer phase obtained for positive $C$ presents a different arrangement from the one obtained for negative $C$, and this is due to the excess of charge located at the center of each IPC. For example, for $C=4$ and $\eta=0.235$ [Fig. 4(h)] the IPCs are arranged as a triangular lattice, but with many defects due to the small value of the potential energy when compared to the kinetic energy. In Fig. 5(b) the average angle distribution for $C=4$ and different area fractions is presented. The peaks around $\phi=60^{\circ}, 120^{\circ}$, and $180^{\circ}$ indicate a triangular lattice structure, which is clearly obtained for low area fraction $(\eta=0.078)$. The triangular lattice structure becomes distorted for larger area fractions due to the tendency to form linear clusters for $C=4$ (Fig. 3). For lower temperature $\left(\sim 10^{-6}\right)$ the number of defects is reduced and a more ordered triangular lattice is obtained (not shown). It is interesting to note that the percolated structures in Figs. 4(c) and 4(e) are essentially distinct. The former originates from T-like clusters, while the latter is obtained from linear clusters. The mechanism driving the percolation transition depends on the net charge $C$, as will be discussed later in this paper.

\section{PERCOLATION}

In this section we examine the connectivity properties of the different equilibrium structures. Configurations are percolated when, accounting for periodic boundary conditions, one of the clusters forms a percolating path [49], i.e., the cluster touches at least two opposite sides of the simulation box. In order to identify the critical area fraction for percolation, we calculate 

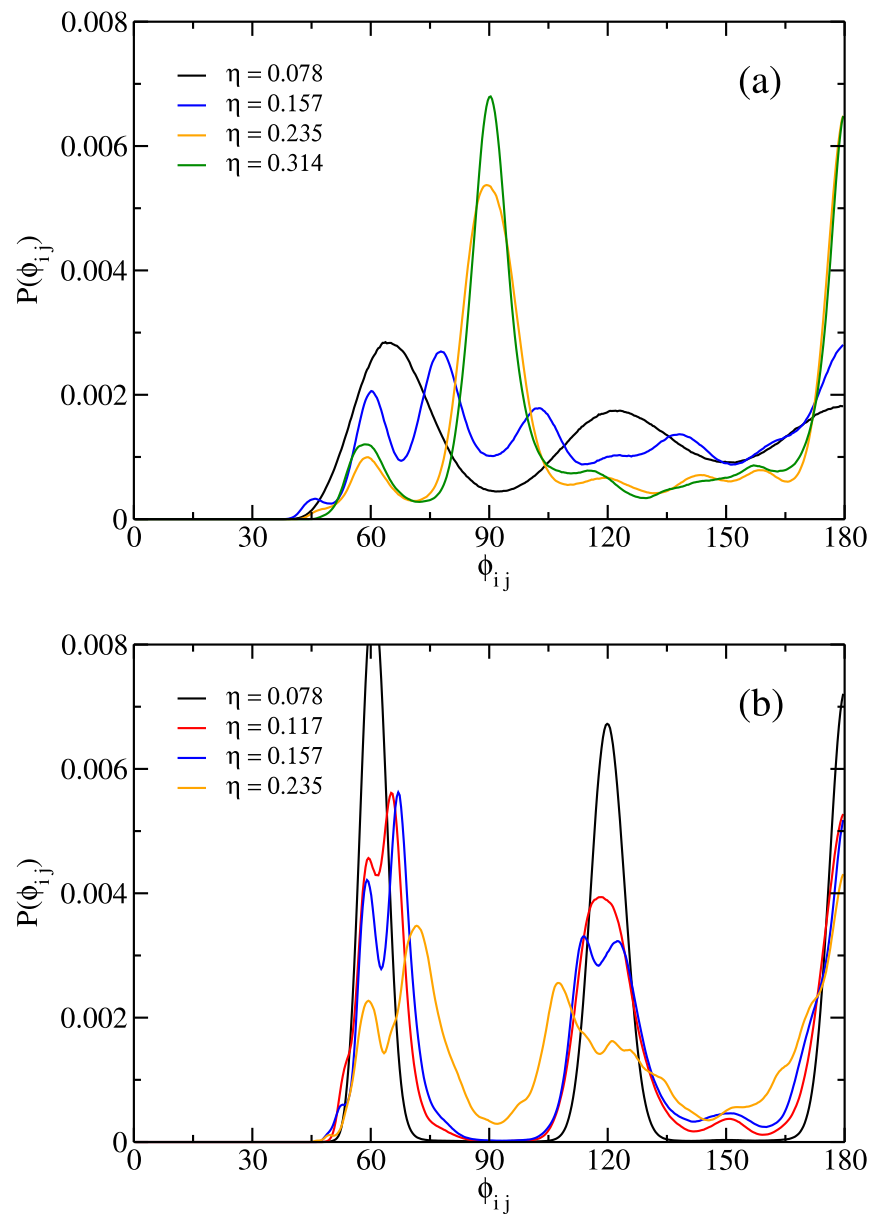

FIG. 5. Distribution of the triplet angle $\phi_{i, j}$ defined in Eq. (6) [see Fig. 1(a)]. For different area fraction and net charge (a) $C=-1$ and (b) $C=4$. In both cases $P\left(\phi_{i j}\right)$ is a normalized distribution.

the average size of the largest cluster $S_{\max }$ as a function of $\eta$. Percolation happens when $S_{\max }$ is at least $50 \%$ of the size of the whole system (Fig. 6) [50,51]. The lowest percolation density is found when the net charge is $C=0$, and this is a consequence of the purely attractive pair-interaction potential in this case, which facilitates the attraction of all particles to the same cluster.

For $C \leqslant-1$ the system does not percolate in the considered $\eta$ interval. The total energy per particle is repulsive for any value of $\eta$, and only monomers are observed. For $C>1$ the critical area fraction for percolation increases with increasing $C$. In this case, linear clusters are observed. We find that the repulsive energy among those clusters overcomes the attractive energy between IPCs in the case of linear clusters. As a consequence, the total energy per particle is repulsive and the average cluster size decreases, which explains why the percolation threshold occurs for higher area fractions (Fig. 6). Such a behavior is opposite to that observed recently by Valadez-Perez et al. [52] for a system with isotropic Yukawa pair-interaction potential.

In order to study percolated structures in more detail we also consider the pair connectedness function $g_{\text {conn }}(r)$, which is defined as the conditional probability of finding a particle at a distance $r$ from a particle located at the origin,

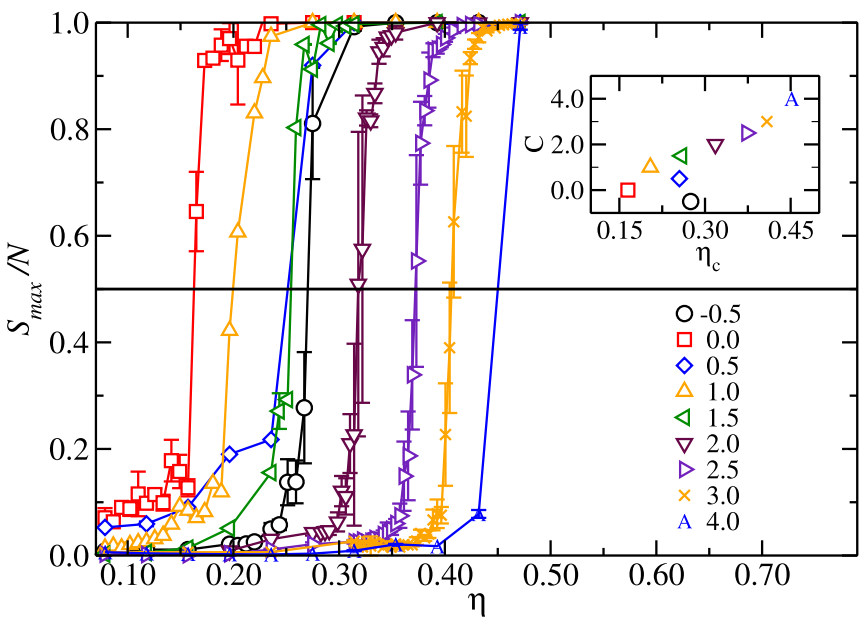

FIG. 6. The average size of the largest cluster $S_{\max }$ as a function of the area fraction for different values of the effective charge $C$. The horizontal solid line represents the percolation threshold defined as $S_{\max } / N=0.50$. The inset shows net charge $(C)$ at which percolation occurs at a given area fraction $\left(\eta_{c}\right)$.

connected via a sequence of bonds, i.e., within the same cluster [35]. In Fig. 7(a), $g_{\text {conn }}(r)$ is shown for $C=3$ and different area fractions, while in Fig. 7(b) $g_{\text {conn }}(r)$ is presented for $\eta=0.235$ and distinct net charges. When an infinite large cluster is present $g_{\text {conn }}(r)$ remains finite on every length scale, as shown in Fig. 7(a) for $\eta>0.43$. On the other hand, the usual radial distribution function $g(r)$ is not sensitive to the percolation transition [see inset in Fig. 7(a)]. Note that $g(r)$ for $\eta=0.314, \eta=0.392$, and $\eta=0.431$ (percolated) exhibits the same general behavior, while $g_{\text {conn }}(r)$ expresses perfectly the percolated case. For $C=3$ and low area fraction $(\eta=0.078)$ there is no clustering and the IPCs self-organize as monomers in a triangular structure with no orientational order (for $T / T_{0}=10^{-3}$ ), similar to the one observed experimentally by Peng et al. [4]. For larger area fraction (e.g., $\eta=0.235$ ), the collective behavior becomes more complex, because the attractive part of the interaction (note that the pair-interaction potential for $C=3$ is purely repulsive) becomes important, resulting in clusterlike structures [Fig. 4(g)]. For larger net charge (e.g., $C=4$ ) the triangular structure of monomers is observed as the ground state for larger area fractions because the repulsive interaction between IPCs starts to dominate.

In Fig. 3 we observe percolated configurations for $C>-1$ and $\eta \gtrsim 0.17$. For high enough area fractions the percolated regions of the phase diagram separate configurations of monomers, which are a consequence of the repulsive interaction due to large negative $(C<-1)$ or large positive net charges. As already stated, the different configurations observed for a given $|C|$ (cases $-C$ and $C$ ) is due to the nonhomogeneous charge distribution in each IPC. Since the negative patches are at the surface of each IPC, a dominant repulsive interaction is observed for lower absolute value of the negative net charge than in the case of positive net charge. We find an interesting reentrant percolation between cluster phases for fixed $\eta$. This is shown in Fig. 7(b), where the pair connectedness function $g_{\text {conn }}(r)$ is presented for fixed area fraction $\eta=0.235$ and distinct net charges. 

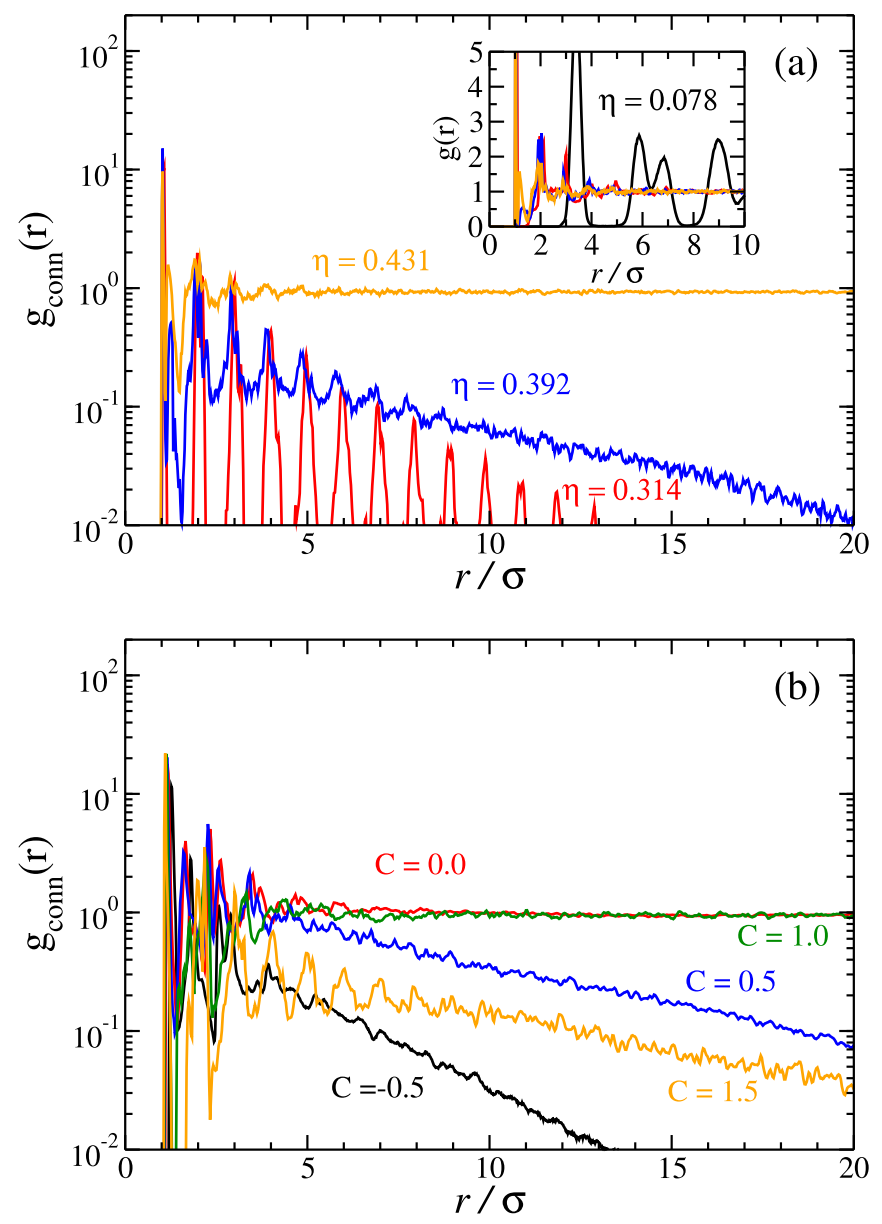

FIG. 7. Pair connectedness function $g_{\text {conn }}(r)$ : (a) for net charge $C=3$ and different area fraction $\eta$ [the corresponding radial distribution function $g(r)$ is shown in the inset] and (b) for fixed area fraction $\eta=0.235$ and different net charge $C$.

For $C=0$ and $C=1$ we find percolated configurations while for $C=-0.5, C=0.5$, and $C>1$ nonpercolated configurations are observed. A reentrant percolation locus in a three-dimensional (3D) system of particles with competitive interaction potential was observed previously as a function of temperature [35]. Here, the system exhibits a different mechanism to induce the reentrant percolation locus, which is found for fixed temperature and density, by changing the net charge of each IPC. The reentrant percolation with respect to the effective electrostatic interaction should be observable since it is experimentally possible to change the charge density in colloids [53] or in proteins [39]. Patchy particles with different materials [4] may result in regions with distinct charges over the colloid.

\section{CLUSTER STRUCTURE}

In the following we study those structures in the phase diagram characterized as cluster phases (symbols $\bigcirc, \square, \triangleleft$ in Figs. 3 and 4) through the cluster size distribution $n(s)$ and the radius of gyration $R_{g}$, both as a function of the cluster size $s$. The former is obtained simply by counting the number of clusters with $s$ components averaged over time. As pointed out
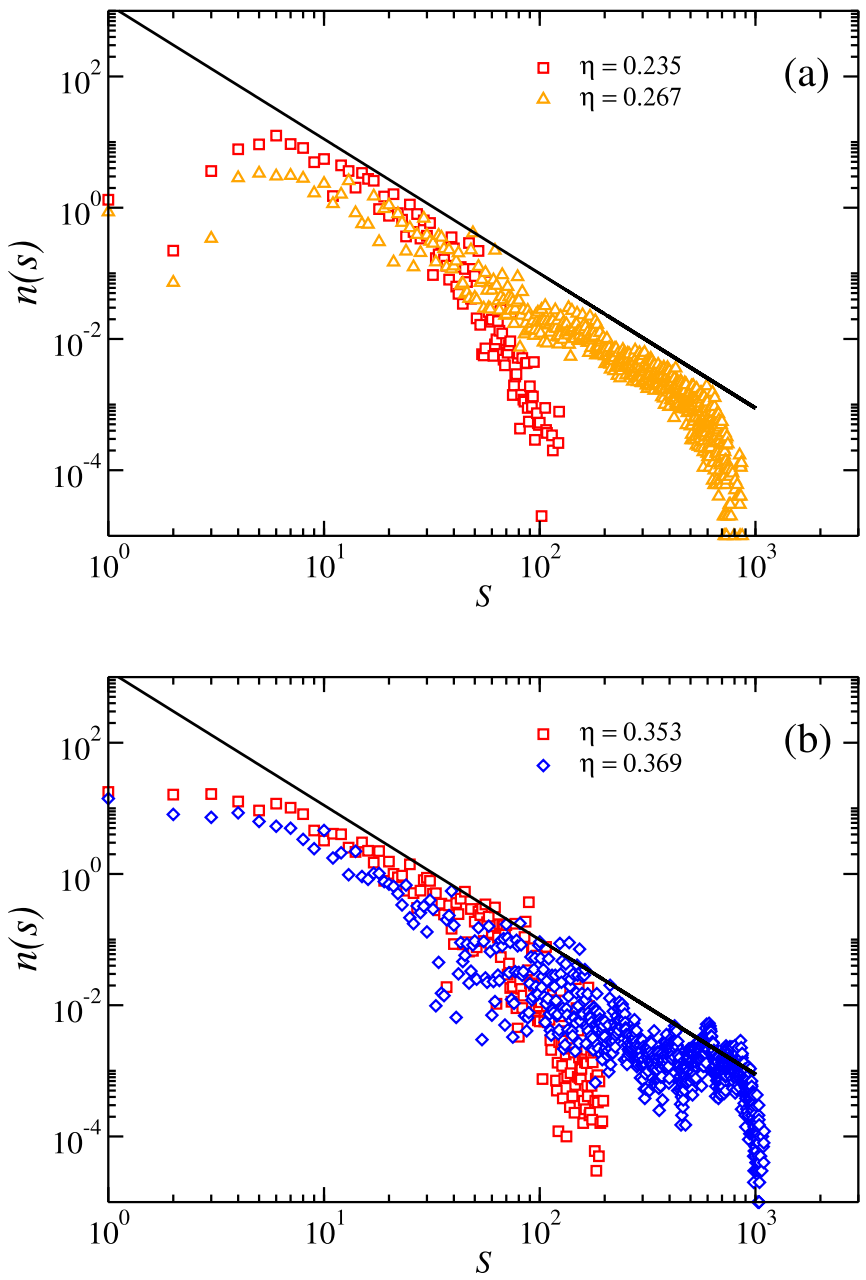

FIG. 8. Cluster size distribution $n_{s}$ for several values of area fraction $\eta$ for net charge (a) $C=-0.5$ and (b) $C=2.5$. In each panel, the solid line represents the function $n_{s} \sim s^{-2.05}$.

in Sec. II, a given particle becomes part of a cluster when its distance from a neighboring particle of the cluster is $r \lesssim 1.3 \sigma$.

The cluster size distribution for different area fractions $\eta$ and representative cases with net charges $C=-0.5$ and $C=2.5$ is presented in Figs. 8(a) and 8(b), respectively. In general, $n(s)$ decreases with increasing cluster size, and for a high enough $\eta$ we observe a percolated structure. In general, close to the percolation transition the $n(s)$ curves develop a power-law dependence, $n(s) \sim s^{\tau}$, with exponent $\tau \approx-2.05$ which is related to random percolation predicted for $2 \mathrm{D}$ ( $\tau=-187 / 91=-2.05$ ) [54], but with distinct percolation mechanisms for different net charges. For $C=-0.5$ the average energy per particle is nearly zero, which means that the bond interaction is unimportant. As a consequence, close to percolation the cluster size distribution behaves as that found in a random percolated system, where an increase of the cluster size occurs due to the aggregation of particles in an aleatory way. Far from the critical area fraction for percolation, only clusters of size $s \leqslant 20$ are observed [ $n(s) \gtrsim 1]$. Such behavior is understood by taking into account the average energy per particle as a function of the cluster size $s$. We find a minimum for $s=3(\eta=0.157)$ and $s=4(\eta=0.235)$. 
For $C=2.5$ linear clusters are observed. The cluster size distribution is also significant for $s \leqslant 20$, but with cluster sizes equally distributed in this interval. For large enough $\eta$ the $n(s)$ curves exhibit a peak for $s \approx 1000$ which is associated to the percolated structure and which is indicative for a gel phase [55]. Such behavior is observed in all cases with no significant bond interaction $(E / N \approx 0)$ or with repulsive interaction $(E / N>0)$.

Now we address the size of the clusters by studying the dependence of the radius of gyration on the cluster size for IPCs with distinct net charges $C$. The radius of gyration $R_{g}(s)$ of a cluster with $s$ particles is defined as

$$
R_{g}(s)=\sqrt{\left\langle\frac{1}{2 s^{2}} \sum_{i, j, i \neq j}\left|r_{i}-r_{j}\right|^{2}\right\rangle_{s}}
$$

where $\langle\cdots\rangle_{s}$ stands for the average over clusters with the same size. The radius of gyration is an interesting quantity since it can be determined experimentally, allowing a direct test for theoretical model systems. In addition, the shape of the clusters can be highlighted from the fractal dimension $d_{f}$ which is obtained from the curves $R_{g} \times s$ through the relation $\left\langle R_{g}(s)\right\rangle \sim s^{1 / d_{f}}$ [49].

In Fig. 9 the radius of gyration as a function of the cluster size is shown for distinct values of the net charge and area fraction. The bond lifetime is very long because of the small temperature $\left(T / T_{0}=10^{-3}\right)$ and the formed clusters remain well defined over time. For $C=-0.5$ the total energy per particle is $E / N \approx 0$ and it changes only slightly as the area fraction increases. This means that the bond interaction is not the main mechanism that determines the formation of the cluster structure. As a consequence, the $R_{g} \times s$ curves are almost independent of the area fraction. As stated before, the random percolated regime in this case is due to the aleatory aggregation of particles in the clusters.

For $C=2.5$ the pair-interaction potential is repulsive with its minimum value obtained when the angle $\theta$ between the main axis of neighbor IPCs is $\theta=0^{\circ}$ (linear bond; see Fig. 1). Such a bond is the main one observed in the many-body case, where the total energy is also repulsive $(E / N>0)$ and it increases with increasing area fraction. The linear bonds $\left(\theta=0^{\circ}\right.$ and $\phi \approx 135^{\circ}$ ) lead to the formation of linear clusters. The linear growth of the clusters for low area fraction is observed in Fig. 9(b) for $\eta \lesssim 0.314$. For larger area fractions the linear clusters start to merge one after another and these larger clusters now are characterized by a different growth regime. For example, for $\eta=0.369$ the radius of gyration exhibits two distinct growth regimes with a clear crossover between them. For small clusters $(s \lesssim 20)$ a more linear regime is observed with $d_{f} \approx 1$, while for the ones with size $s \gtrsim 50$ their structures are characterized by pieces of linear clusters connected to each other through branching points (linear ramified clusters). The branches are connected in a random way in order that the radius of gyration of such clusters scales with the size $s$ with $d_{f} \approx 91 / 48$ which characterizes the random percolation regime in $2 \mathrm{D}$. The mechanism for the formation of ramified linear clusters described here is observed for the cases with $C \geqslant 1$, and it is analogous to the 3D network structures obtained with Bernal spirals in 3D
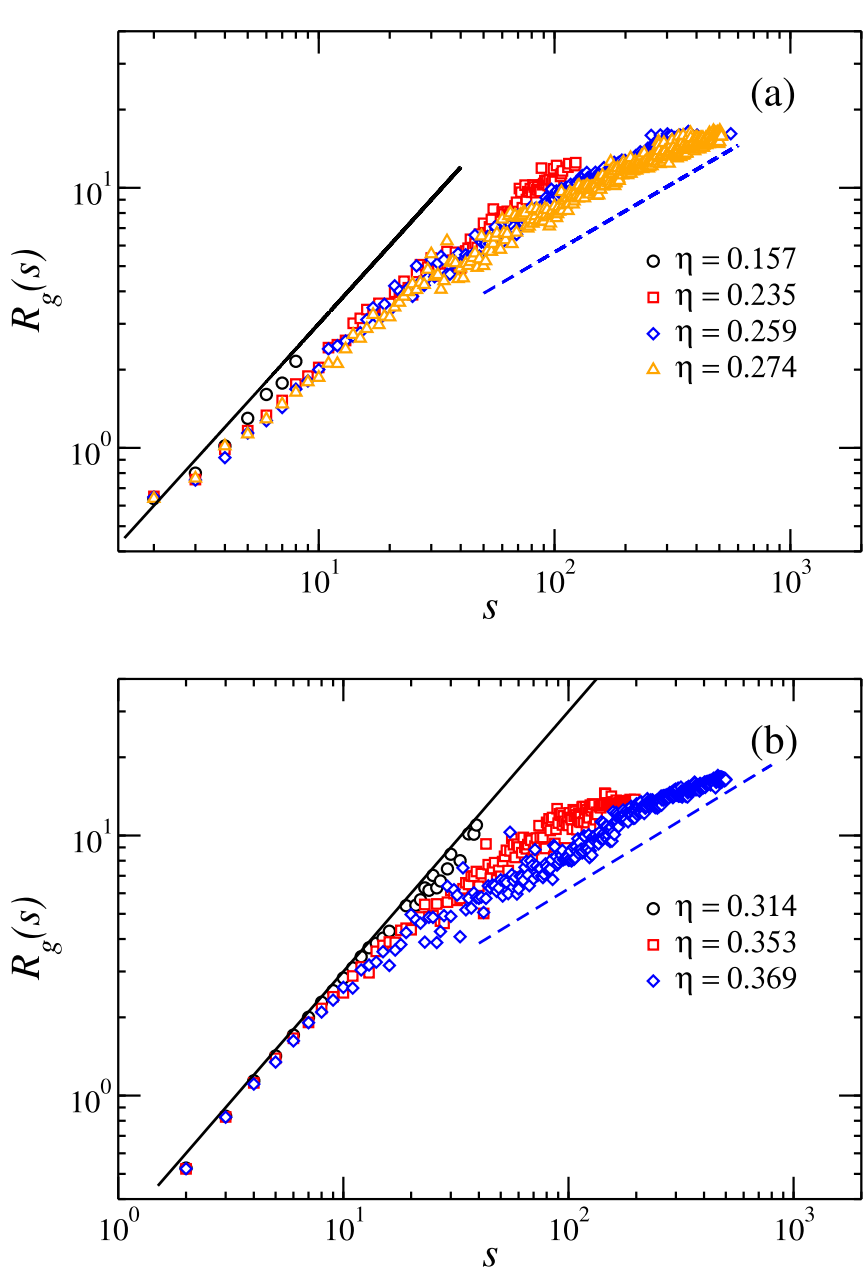

FIG. 9. Size dependence of the cluster gyration radius for several area fractions and net charge (a) $C=-0.5$ and (b) $C=2.5$. The black (solid) line corresponds to $d_{f}=1$, while the blue (dashed) line corresponds to $d_{f}=91 / 48$ in the relation $\left\langle R_{g}(s)\right\rangle \sim s^{1 / d_{f}}$.

[35]. As compared to the case with $C=-0.5(E / N \approx 0)$, the distinct mechanism observed in the case $C=2.5(E / N>0)$ is due to the stronger interaction between IPCs that induces the formation of linear bonds.

In Fig. 10 we present the average distribution of the angle between the main axis of neighbor IPCs for $C=-0.5$ and $C=2.5$ and distinct area fractions. This quantity helps us to better understand the microstructure of the obtained configurations. As mentioned previously, we observe three different types of bonds (Fig. 1). For $C \lesssim-0.5$ the total energy per particle is repulsive for area fraction $\eta \gtrsim 0.08$, but the microstructure of the system changes with $\eta$. For a very diluted system (low $\eta$ ) the small clusters are mostly formed by pairs of IPCs connected through T-like bonds. Note that the pair-interaction potential for $C=-0.5$ presents a very shallow global minimum at $r \approx 1.2 \sigma$ and a very short repulsive barrier that separates the global minimum from the minimum at $r \rightarrow \infty$ (Fig. 2), which favors the formation of small stable clusters consisting of few particles for low $\eta$. For $\eta=0.117$ and $\eta=0.157$ small clusters with typically $N=3-5$ particles favor the formation of $\mathrm{V}$-like bonds (with $60^{\circ} \lesssim \theta \lesssim 90^{\circ}$ ). For larger area fraction $\eta$ (close to percolation) the larger and 

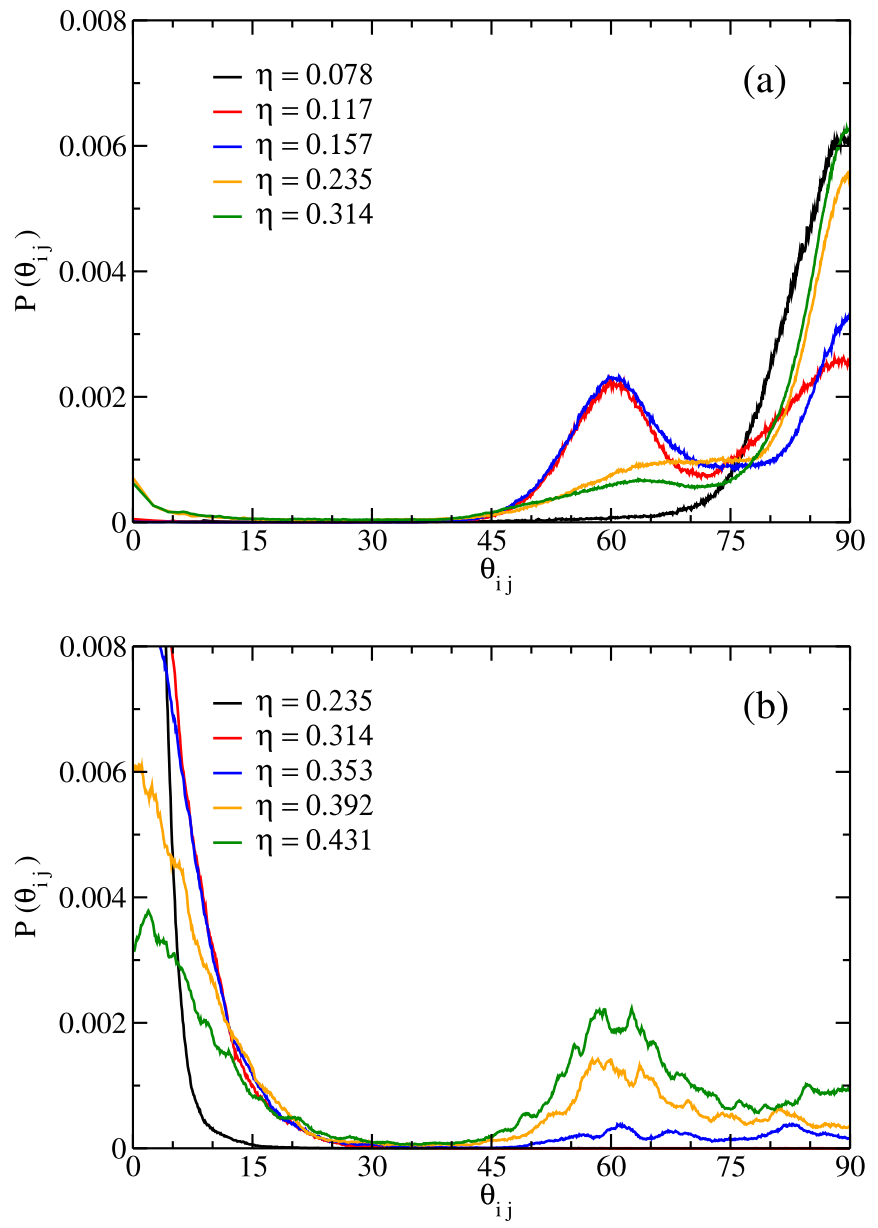

FIG. 10. Average angle between the main axis of neighbor bonded patchy particles for different area fractions and net charge (a) $C=-0.5$ and (b) $C=2.5$. In both cases $P\left(\theta_{i j}\right)$ is a normalized distribution.

more rounded clusters favor the formation of T-like bonds [see Fig. 10(a), $\eta=0.314$ ].

For $C \geqslant 1.5$ the total energy per particle is repulsive for any $\eta$, but the microstructure of the clusters is essentially different as a consequence of the nonhomogeneous charge distribution. For low values of $\eta$, the bonds between IPCs are mostly linear resulting in linear clusters. The repulsive energy between those linear clusters overcomes the attractive interaction between the IPCs in the clusters. As the area fraction increases the linear clusters start to merge one after another with angles distributed in the interval $45^{\circ}<\theta \lesssim 90^{\circ}$. However, in this case the linear bond is still the main feature of all self-assembled configurations. These features are illustrated in Fig. 10(b) for $C=2.5$.

\section{INTERACTION RANGE}

Now we discuss the effects of the range of the pair interaction potential on the self-assembled configurations. Experimentally, the range of the interaction in colloidal systems can be modified, e.g., by changing the $p \mathrm{H}$ of the solvent that the particles are immersed in. In this section, we illustrate how the self-assembled configurations are modified
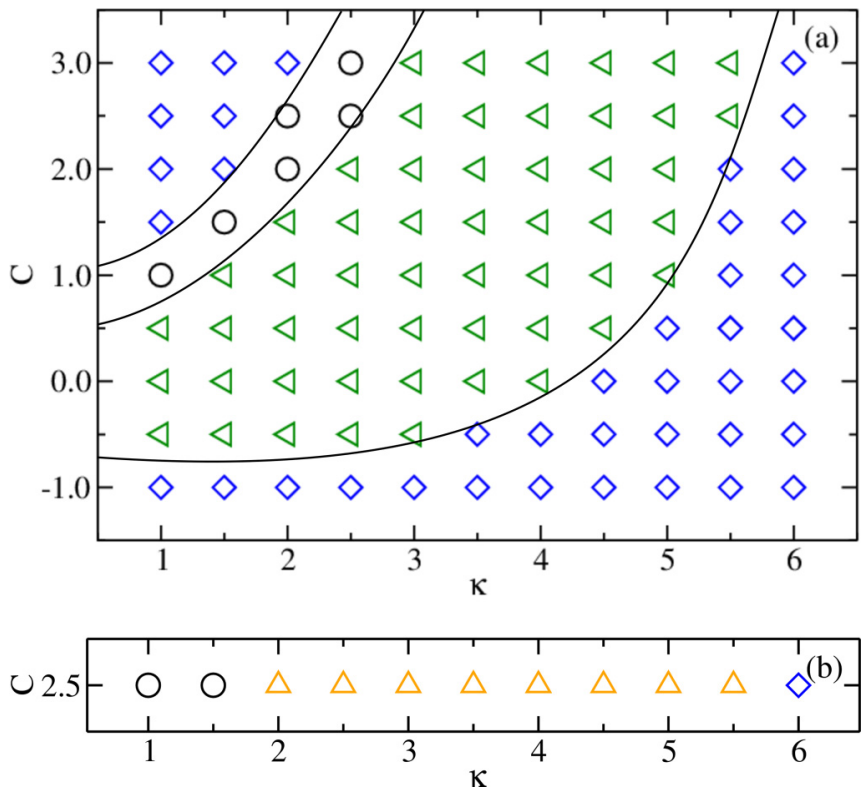

FIG. 11. Structural phase diagram in the plane of screening parameter $(\kappa)$ and net charge $(C)$ for (a) $\eta=0.078$ and (b) $\eta=0.235$. Symbols represent different structures: $\diamond$, monomers; $\triangleleft$, mixed clusters; ○, linear clusters; and $\Delta$, percolated clusters. Solid lines are a guide to the eyes that separate the different phases.

as a function of the net charge $C$ and screening parameter of the pair-interaction potential $\kappa$. The dimensionless temperature is kept constant at $T / T_{0}=10^{-3}$. The results for the area fractions $\eta=0.078$ and $\eta=0.235$ are presented in Fig. 11 .

In general, for large enough $\kappa$ (short-range interaction) the IPCs self-assemble as a set of monomers, which may or may not present an ordered arrangement according to the values of $T, \eta$, and $C$. For $\eta=0.078$ [Fig. 11(a)] a fluid phase is found for $\kappa \geqslant 6$ due to the small area fraction and the relatively large temperature (in comparison to the interaction among IPCs). The total interaction energy is almost zero and the system behaves approximately as an ideal gas. The monomer phase is also observed for small $\kappa \leqslant 3$ and sufficiently large $|C|$, but in this limit it is due to the strong repulsion between IPCs. As a consequence of such a strong interaction, triangular or squared ordered arrangements can be observed, as mentioned previously for the case with $\kappa=1$. The triangular lattice is obtained for positive and negative (low $\eta$ ) $C$, while the square lattice is obtained only for negative $C$. Such an asymmetry in the self-assembled configurations is a consequence of the nonhomogeneous charge distribution over the IPC. For intermediate values of $\kappa$ and $C$, linear and mixed clusters are found as a consequence of the interaction among the nonhomogeneously charged IPC.

For large area fraction, percolated phases become possible. For distinct charges we always find a random percolation regime, as shown previously for $\kappa=1$, but the leading mechanism of percolation depends on $(C, \kappa)$. For example, for $\eta=0.235$ and $C=2.5$ [Fig. 11(b)] the percolated phases observed for $\kappa \lesssim 3$ originate from the random merging between linear clusters, which are characterized by linear bonds. On the other hand, for larger values of the screening parameter (e.g., $\kappa \approx 5$ ) the percolated phase arises from the 
aggregation among more rounded clusters (mixed cluster phase). In this case, the IPCs are mainly connected to each other through T-like bonds, which causes an increase in the average number of neighbors per particle.

\section{CONCLUSIONS}

We presented a systematic numerical study of the minimum energy configurations of a 2D system of IPCs. Each IPC is modeled as a disk with central positive pointlike charge $Q$ and two patches, each with pointlike charge $-q$ in diametral opposite positions, located at the surface. The diametral line connecting the two patches defines the main axis of a given IPC. The many-body system was studied as a function of the net charge $C=Q-2|q|$ of each IPC and the area fraction $\eta$. The lowest energy configurations consisting of monomers, linear clusters, ramified-linear clusters, mixed clusters, and percolated configurations were summarized in a $C-\eta$ phase diagram. The effects of the range of the interaction potential were also addressed for some specific densities.

Due to the nonhomogeneous charge distribution over the colloids, there is no symmetry in the pair-interaction potential with respect to the sign of the net charge. As a consequence, for the same absolute value $|C|$, a configuration observed for a given negative $C$ is different from the one found for positive $C$. For sufficient large value of $|C|$ crystal-like arrangements of monomers are found for both positive and negative net charges. Interestingly, due to the asymmetry in the charge distribution, it is possible that the system of IPCs with high enough negative $C$ self-assemble in a square lattice. On the other hand, for IPCs with high enough positive net charge the effective repulsive pair interaction has higher radial symmetry (positive charge at the center of each IPC) and the observed configuration turns out to be a triangular lattice.

The shape and the size of the clusters were also investigated for different net charges and area fractions $(\kappa=1)$. In general, for $|C|<1$ the IPCs self-assemble in more rounded clusters mainly characterized by T-like bonds (mixed clusters), while for $|C| \geqslant 1$ the self-assembled configurations depend on the sign of the net charge. For negative $C<-1$, the monomer phase is always found for any area fraction, while for positive $C$ the monomer phase is observed for small area fraction. By increasing the area fraction, linear clusters and ramified linear clusters are observed, before the percolated phase is observed for large enough area fraction. We also studied how the microstructure of the clusters depends on $C$ by calculating the average angle between bonded neighbor particles. In general, the clusters exhibit three types of bonds (linear, T-like, and V-like bonds) which depends on the net charge and area fraction. For $\kappa=1$, linear bonds are found for $C \geqslant 1$, while T-like bonds are observed only in the interval $|C| \lesssim 0.5$. For $\kappa \neq 1$, linear bonds are still observed for $C \geqslant 1$, but T-like bonds becomes possible for larger values of $C$ in the low area fraction limit.

A reentrant transition between percolated and nonpercolated configurations in a finite interval of area fraction and net charge was observed. The random percolation transition observed from our study is driven through distinct mechanisms which depends on the net charge of the IPC. For example, for $C>1$ percolation occurs through the random aggregation of linear clusters, while for $-1<C<1$ the percolation transition is the result of the aleatory aggregation of IPCs in more rounded clusters. For a given area fraction the reentrant percolation transition is driven by changes in the net charge of the colloids.

Self-assembled configurations are becoming tools in the development of new materials. From our model we observed distinct self-assembled structures which depend on the different model parameters $(C, \kappa$, and $\eta)$. For example, we found that square or triangular lattices may be obtained according to the net charge of the IPCs. The different self-assembled crystal structures will lead to different interference patterns of light which are of great importance in applications of photonic crystals. An interesting extension of the present work is the combination of the present anisotropic interaction with different external parameters (temperature and/or pressure), and to consider the effect of the number of charged patches and its distribution (regular or irregular) over the surface of the particles on the self-assembled configurations.

\section{ACKNOWLEDGMENTS}

This work was supported by the Brazilian agencies FUNCAP, CAPES, the program Science without borders, and CNPq (Project 400748/2013-4), and by the joint CNPq-FWO bilateral project and the Flemish Science Foundation (FWOV1).
[1] A. Yethiraj and A. van Blaaderen, Nature (London) 421, 513 (2003).

[2] U. Gasser, E. R. Weeks, A. Schofield, P. N. Pusey, and D. A. Weitz, Science 292, 258 (2001).

[3] V. J. Anderson and H. N. W. Lekkerkerker, Nature (London) 416, 811 (2002).

[4] B. Peng, A. van Blaaderen, and A. Imhof, ACS Appl. Mater. Interfaces 5, 4277 (2013).

[5] S. C. Glotzer and M. J. Solomon, Nat. Mater. 6, 557 (2007).

[6] E. Duguet, A. Desert, A. Perro, and S. Ravaine, Chem. Soc. Rev. 40, 941 (2011).

[7] L. Manna, E. C. Scher, and A. P. Alivisatos, J. Am. Chem. Soc. 122, 12700 (2000).
[8] Y. Y. Yu, S. S. Chang, C. L. Lee, and C. R. Wang, J. Phys. Chem. B 101, 6661 (1997).

[9] A. B. Pawar and I. Kretzschmar, Langmuir 25, 9057 (2009).

[10] K. H. Roh, D. C. Martin, and J. Lahann, J. Am. Chem. Soc. 128, 6796 (2006).

[11] A. F. Demirörs, J. C. P. Stiefelhagen, T. Vissers, F. Smallenburg, M. Dijkstra, A. Imhof, and A. van Blaaderen, Phys. Rev. X 5, 021012 (2015).

[12] A. B. Pawar and I. Kretzschmar, Macromol. Rapid Commun. 31, 150 (2010).

[13] E. Bianchi, R. Blaak, and C. N. Likos, Phys. Chem. Chem. Phys. 13, 6397 (2011). 
[14] E. G. Noya, C. Vega, J. P. K. Doye, and A. A. Louis, J. Chem. Phys. 127, 054501 (2007).

[15] S. Deka, K. Miszta, D. Dorfs, A. Genovese, G. Bertoni, and L. Manna, Nano Lett. 10, 3770 (2010).

[16] S. Sacanna, W. T. M. Irvine, L. Rossi, and D. J. Pine, Soft Matter 7, 1631 (2011).

[17] R. P. Sear, J. Chem. Phys. 111, 4800 (1999).

[18] X. Song, Phys. Rev. E 66, 011909 (2002).

[19] N. Kern and D. Frenkel, J. Chem. Phys. 118, 9882 (2003).

[20] J. Chang, A. M. Lenhoff, and S. I. Sandler, J. Phys. Chem. B 109, 19507 (2005).

[21] J. Drenth, Principles of Protein X-Ray Crystallography, 3rd ed. (Springer-Verlag, New York, 2007).

[22] P. R. ten Wolde and D. Frenkel, Science 277, 1975 (1997).

[23] Y. S. Cho, G. R. Yi, J. M. Lim, S. H. Kim, V. N. Manoharan, D. J. Pine, and S. M. Yang, J. Am. Chem. Soc. 127, 15968 (2005).

[24] Y. S. Cho, G. R. Yi, S. H. Kim, D. J. Pine, and S. M. Yang, Chem. Mater. 17, 5006 (2005).

[25] G. R. Yi, V. N. Manoharan, E. Michel, M. T. Elsesser, S. M. Yang, and D. J. Pine, Adv. Mater. 16, 1204 (2004).

[26] V. N. Manoharan, M. T. Elsesser, and D. J. Pine, Science 301, 483 (2003).

[27] Q. Chen, S. Chul Bae, and S. Granick, Nature (London) 469 381 (2011).

[28] A. Stradner, H. Sedgwick, F. Cardinaux, W. C. K. Poon, S. U. Egelhaaf, and P. Schurtenberger, Nature (London) 432, 492 (2004).

[29] A. I. Campbell, V. J. Anderson, J. S. van Duijneveldt, and P. Bartlett, Phys. Rev. Lett. 94, 208301 (2005).

[30] Y. Liu, W. R. Chen, and S. H. Chen, J. Chem. Phys. 122, 044507 (2005).

[31] J. Wu, Y. Liu, W.-R. Chen, J. Cao, and S. -H. Chen, Phys. Rev. E 70, 050401 (2004).

[32] J. Groenewold and W. K. Kegel, J. Phys.: Condens. Matter 16, S4877 (2004).

[33] S. Mossa, F. Sciortino, P. Tartaglia, and E. Zaccarelli, Langmuir 20, 10756 (2004).
[34] F. Sciortino, S. Mossa, E. Zaccarelli, and P. Tartaglia, Phys. Rev. Lett. 93, 055701 (2004).

[35] F. Sciortino, P. Tartaglia, and E. Zaccarelli, J. Phys. Chem. B 109, 21942 (2005).

[36] C. B. Muratov, Phys. Rev. E 66, 066108 (2002).

[37] M. E. Cates and S. J. Candau, J. Phys.: Condens. Matter 2, 6869 (1990).

[38] H. Sedgwick, K. Kroy, A. Salonen, M. B. Robertson, S. U. Egelhaaf, and W. C. K. Poon, Eur. Phys. J. E 16, 77 (2005).

[39] Rikkert J. Nap, A. Lošdorfer Božič, and R. Podgornik, Biophys. J. 107, 1970 (2014).

[40] E. Bianchi, G. Kahl, and C. N. Likos, Soft Matter 7, 8313 (2011).

[41] D. El Masri, P. van Oostrum, F. Smallenburg, T. Vissers, A. Imhof, M. Dijkstra, and A. van Blaaderen, Soft Matter 7, 3462 (2011).

[42] E. G. Noya, I. Kolovos, G. Doppelbauer, G. Kahl, and E. Bianchi, Soft Matter 10, 8464 (2014).

[43] E. Bianchi, C. N. Likos, and G. Kahl, ACS Nano 7, 4657 (2013).

[44] E. Bianchi, C. N. Likos, and G. Kahl, Nano Lett. 14, 3412 (2014).

[45] P. D. J. van Oostrum, M. Hejazifar, C. Niedermayer, and E. Reimhult, J. Phys.: Condens. Matter 27, 234105 (2015).

[46] M. P. Allen and D. J. Tildesley, Computer Simulation of Liquids (Oxford University Press, Oxford, 1987).

[47] L. Bonsall and A. A. Maradudin, Phys. Rev. B 15, 1959 (1977).

[48] G. Malescio and G. Pellicane, Nat. Mater. 2, 97 (2003).

[49] D. Stauffer and A. Aharony, Introduction to Percolation Theory (Taylor Francis Group, Philadelphia, PA, 1994).

[50] Y. Liu and R. B. Pandey, J. Chem. Phys. 105, 825 (1996).

[51] R. Chelakkot and T. Gruhn, Soft Matter 8, 11746 (2012).

[52] N. E. Valadez-Perez, R. Castaneda-Priego, and Y. Liu, RSC Adv. 3, 25110 (2013).

[53] A. F. C. Campos, F. A. Tourinho, G. J. da Silva, M. C. F. L. Lara, and J. Depeyrot, Eur. Phys. J. E 6, 29 (2001).

[54] M. Rubinstein and R. H. Colby, Polymer Physics (Oxford University Press, New York, 2003).

[55] P. Douglas Godfrin, N. E. Valadez-Perez, R. Castaneda-Priego, N. J. Wagner, and Y. Liu, Soft Matter 10, 5061 (2014). 